\title{
○ \\ Reduced $\alpha$-defensin expression is associated with inflammation and not NOD2 mutation status in ileal Crohn's disease
}

\author{
L A Simms, ${ }^{1,2}$ J D Doecke, ${ }^{2}$ M D Walsh, ${ }^{2}$ N Huang, ${ }^{1,2}$ E V Fowler, ${ }^{1,3}$ G L Radford-Smith ${ }^{1,4}$
}

${ }^{1}$ Inflammatory Bowel Disease Laboratory, Royal Brisbane and Women's Hospital Foundation, Brisbane, Australia;

${ }^{2}$ Queensland Institute of Medical Research, Brisbane, Australia; ${ }^{3}$ School of Medicine The University of Queensland, Brisbane; ${ }^{4}$ Department of Gastroenterology, Royal Brisbane and Women's Hospital, Brisbane, Australia

Correspondence to: Lisa A Simms, Inflammatory Bowel Disease Laboratory, Queensland Institute of Medical Research, Brisbane 4029, Australia; Lisa.Simms@ qimr.edu.au

Revised 13 February 2008 Accepted 19 February 2008 Published Online First

27 February 2008

\begin{abstract}
Background and aims: Reduced ileal Paneth cell $\alpha$ defensin expression has been reported to be associated with Crohn's disease, especially in patients carrying NOD2 mutations. The aim of this study was to independently assess whether NOD2, $\alpha$-defensins and Crohn's disease are linked.

Methods: Using quantitative real-time polymerase chain reaction (RT-PCR), we measured the mRNA expression levels of key Paneth cell antimicrobial peptides (DEFA5, DEFA6, LYZ, PLA2G2A), inflammatory cytokines [interkelukin 6 (IL6) and IL8], and a marker of epithelial cell content, villin (VIL1) in 106 samples from both affected ileum (inflamed Crohn's disease cases, $n=44$ ) and unaffected ileum (non-inflamed; Crohn's disease cases, $n=51$ and controls, $n=11$ ). Anti-human defensin 5 (HD5) and haematoxylin/eosin immunohistochemical staining was performed on parallel sections from NOD2 wild-type and NOD2 mutant ileal Crohn's disease tissue.
\end{abstract}

Results: In Crohn's disease patients, DEFA5 and DEFA6 mRNA expression levels were 1.9- and 2.2-fold lower, respectively, in histologically confirmed inflamed ileal mucosa after adjustment for confounders (DEFA5, $p<0.001$; DEFA6, $p=0.001$ ). In contrast to previous studies, we found no significant association between $\alpha$ defensin expression and NOD2 genotype. HD-5 protein data supports these RNA findings. The reduction in HD- 5 protein expression appears due to surface epithelial cell loss and reduced Paneth cell numbers as a consequence of tissue damage.

Conclusions: Reduction in $\alpha$-defensin expression is independent of NOD2 status and is due to loss of surface epithelium as a consequence of inflammatory changes rather than being the inciting event prior to inflammation in ileal Crohn's disease.

Crohn's disease is one of two major forms of inflammatory bowel disease (IBD), and is a chronic, debilitating disorder with increasing incidence and prevalence globally. ${ }^{1-3}$ Inflammation in Crohn's disease most commonly involves the terminal ileum with up to $75 \%$ of patients having ileal disease with or without colonic involvement. ${ }^{4}$ It is unclear as to why the ileum is so susceptible to Crohn's disease. Relevant features include significantly higher numbers of bacteria compared to the more proximal small bowel, ${ }^{5}$ large amounts of lymphoid tissue including Peyer's patches, and Paneth cells, which produce antimicrobial peptides. ${ }^{6}$

Paneth cells are specialised epithelial cells located at the base of small intestinal crypts. On exposure to bacteria and bacterial antigens, Paneth cells release antimicrobial peptides, including lysozyme
(LYZ), secretory phospholipase $\mathrm{A}_{2}$ (PLA2G2A), and human $\alpha$-defensins 5 and 6 (DEFA5 and DEFA6) with DEFA5 and DEFA6 being the most abundant Paneth cell antimicrobials expressed in the small intestine. The release of these peptides into the crypt lumen is believed to prevent microbial invasion into the crypt microenvironment. ${ }^{8}$ Human DEFA5 is active against several bacterial species, including Listeria monocytogenes, Escherichia coli and Salmonella typhimurium, and against the fungus Candida albicans. ${ }^{9}$ Similarly, murine Paneth cell $\alpha$-defensins, also known as cryptdins, have microbicidal activity against $E$ coli, Staphylococcus aureus and $S$ typhimurium. Murine cryptdins reach concentrations at least 1000 times higher than minimal bactericidal concentrations in vitro, indicating the potential for $\alpha$-defensins to maintain sterility in the crypt and protect the stem cells. ${ }^{11}$ DEFA5 transgenic mice confer marked resistance to oral challenges with $S$ typhimurium ${ }^{12}$ and mice deficient in the $\alpha$-defensin-processing enzyme, matrilysin, are highly susceptible to orally administered bacteria. ${ }^{13}$ Hence, in humans, lower concentrations of $\alpha$-defensins reaching the intestinal crypt could presumably render the crypt susceptible to colonisation by resident microflora.

Paneth cells also express NOD2, an intracellular receptor for muramyl dipeptide, which is derived from the bacterial cell wall component peptidoglycan. ${ }^{14}$ The recognition of bacterial products by NOD2 activates signal transduction pathways via nuclear factor kappa B (NF- $\mathrm{KB})$ as part of the innate immune response. ${ }^{15} 16$ Mutations in the NOD2 gene (R702W, G908R and 1007fs) are strongly associated with ileal location and contribute towards approximately 25\% of Crohn's disease overall and $50 \%$ of pure ileal disease. ${ }^{417}$

It has been reported that patients with ileal Crohn's disease have reduced mRNA expressions of DEFA5 and DEFA6 in affected (inflamed) ileum, compared to unaffected ileum and ileum from healthy controls. This reported reduction in $\alpha$ defensin expression was significantly greater in patients with NOD2 mutations and independent of the degree of inflammation. ${ }^{18}{ }^{19}$ In separate studies investigating interleukin 8 (IL8) responses in Crohn's disease, peripheral blood mononuclear cells from patients with NOD2 mutations show a diminished response to muramyl dipeptide stimulation. ${ }^{20}{ }^{21}$ Here, by carefully sampling inflamed and non-inflamed mucosa, we show that the reduction in DEFA5 and DEFA6 expression in mucosal biopsies from patients with ileal Crohn's disease is not associated with NOD2 status and is 
Table 1 Characteristics of the study groups

\begin{tabular}{|c|c|c|}
\hline & Crohn's disease & Controls \\
\hline \multicolumn{3}{|l|}{ Patient data } \\
\hline No. of subjects & 65 & 11 \\
\hline Mean age (years) & 31.4 (SD 11.4) & 52.7 (SD 17.7) \\
\hline Sex, female, $\mathrm{n}$ & $35(54 \%)$ & $8(73 \%)$ \\
\hline Ileal involvement, $\mathrm{n}$ & $65(100 \%)$ & \\
\hline \multicolumn{3}{|l|}{ Medical treatment } \\
\hline None, $\mathrm{n}$ & $37(57 \%)$ & \\
\hline Treatment, $\mathrm{n}$ & $28(43 \%)$ & \\
\hline Monotherapy, $\mathrm{n}$ & 13 & \\
\hline Immunosuppression, $\mathrm{n}$ & 10 & \\
\hline Steroids, $\mathrm{n}$ & 2 & \\
\hline 5-ASA, n & 1 & \\
\hline Combination therapy, $\mathrm{n}$ & 15 & \\
\hline \multicolumn{3}{|l|}{ Sample data } \\
\hline No. of samples & 95 & 11 \\
\hline \multicolumn{3}{|l|}{ Type } \\
\hline Resection, $\mathrm{n}$ & $21(22 \%)$ & $0(0 \%)$ \\
\hline Biopsy, n & $74(78 \%)$ & $11(100 \%)$ \\
\hline \multicolumn{3}{|l|}{ Histology } \\
\hline Non-inflamed, $\mathrm{n}$ & $51(54 \%)$ & $11(100 \%)$ \\
\hline Inflamed, $\mathrm{n}$ & $44(46 \%)$ & $0(0 \%)$ \\
\hline \multicolumn{3}{|l|}{ NOD2 status } \\
\hline Wild-type, $n$ & $58(61 \%)$ & $8(73 \%)$ \\
\hline Mutant*, n & 37 (39\%) & $3(27 \%)$ \\
\hline
\end{tabular}

*Heterozygous or homozygous for any variant (R702W, G908R, 1007fs).

dependent on inflammatory state. We propose that the decrease in $\alpha$-defensin expression is due to loss of epithelium, secondary to inflammatory changes, rather than being the inciting event prior to inflammation in ileal Crohn's disease.

\section{METHODS}

\section{Patient samples}

Sixty-five Crohn's disease patients and 11 healthy controls were recruited for the study (table1). These were made up of 21 resection specimens obtained from 13 Crohn's disease patients, and 85 sets of colonoscopic biopsy specimens from a consecutive unselected series of 52 Crohn's disease patients and 11 healthy control individuals. We used tissue samples based on inflammatory status and histology as the experimental unit, since duplicate non-inflamed and inflamed samples could only be obtained from a subgroup of patients. Colonoscopy was performed by a single gastroenterologist (G.R.-S.) at the Royal Brisbane and Women's Hospital on patients with a diagnosis of ileal Crohn's disease and from healthy controls undergoing colorectal cancer family history screening. The diagnosis of Crohn's disease was based on standard criteria. ${ }^{22}$ Patients with pure colonic Crohn's disease were excluded from this study. All subjects gave written informed consent. Where possible, from within a $2-\mathrm{cm}^{2}$ area, five pinch biopsy samples were retrieved for RNA and DNA extraction from both affected ileum (inflamed, cases) and unaffected ileum (non-inflamed, cases and controls). Additional biopsy samples from within these same areas were retrieved and placed in formalin for histological examination by a pathologist. Tissue samples obtained after resection were also formalin fixed and paraffin embedded prior to histological examination. Samples for RNA and DNA extraction were immediately snap frozen in dry ice following withdrawal of the endoscope, and then stored at $-80^{\circ} \mathrm{C}$ until use. Medical treatment for the 65 Crohn's disease patients at the time of tissue sampling was as follows: no medical therapy, $\mathrm{n}=37$; monotherapy, $\mathrm{n}=13$; immunosuppression, $\mathrm{n}=10$; steroids, $\mathrm{n}=2$; 5-aminosalicylic acid (5-ASA), $\mathrm{n}=1$; combination therapy, $\mathrm{n}=15$ (immunosuppression, steroids, 5-ASA). None of the patients were receiving antibiotic therapy.

\section{NOD2 genotyping by high-resolution melt curve analysis}

Genotyping of the common disease-predisposing NOD2 variants (R702W, G908R and 1007FS), ${ }^{23}{ }^{24}$ and a marker of the NOD2 haplotype (IVS8 = 158), P268S, ${ }^{25}$ was performed by highresolution melt analysis on a Rotor-Gene 6000 (Corbett Life Science, Sydney, Australia). Briefly, $25 \mathrm{ng}$ of genomic DNA isolated from either peripheral blood or ileal biopsy tissue was amplified using the following primers: P268S, forward 5'-TACCTATGATGGAGCAGAGACGCT-3', reverse 5'-TGAAGAGCTCCTCCAGGCCCA-3'; R702W, forward 5'-GCTGGCTGAGTGCCAGACAT-3', reverse 5'-GATGGAGTGGAAGTGCTTGCG-3'; G908R, 5'-AGAGGGAGGAGGACTGTTAGTTCA-3', reverse 5'-ATCACCCAAGGCTTCAGCCAG-3'; 1007FS, 5'-TTTCCAGGTTGTCCAATAACTGCATCAC -3', reverse 5'-GGGCCTTACCAGACTTCCAGG-3'. Polymerase chain reaction (PCR) was carried out in a $16-\mu$ l reaction volume containing $1 \mu \mathrm{l}$ genomic DNA (25 ng), 0.8× Platinum PCR SuperMix (Invitrogen, California, USA), $300 \mathrm{nmol} / \mathrm{l}$ each primer, $1.25 \mu \mathrm{mol} / 1$ Syto9 green fluorescent nucleic acid dye (Invitrogen) and $\mathrm{H}_{2} \mathrm{O}$. The cycling conditions were one cycle at $95^{\circ} \mathrm{C}$ for $3 \mathrm{~min}, 40$ cycles at $95^{\circ} \mathrm{C}$ for $30 \mathrm{~s}, 57^{\circ} \mathrm{C}$ for $15 \mathrm{~s}, 72^{\circ} \mathrm{C}$ for $20 \mathrm{~s}$, followed by one cycle at $72^{\circ} \mathrm{C}$ for $3 \mathrm{~min}$, one cycle at $50^{\circ} \mathrm{C}$ for $20 \mathrm{~s}$ and a high-resolution melt cycle rising by $0.1^{\circ}$ each step and ramping from 81 to $91^{\circ} \mathrm{C}$ for $\mathrm{P} 268 \mathrm{~S}, 84$ to $94^{\circ} \mathrm{C}$ for $\mathrm{R} 702 \mathrm{~W}, 78$ to $88^{\circ} \mathrm{C}$ for G908R and 77 to $87^{\circ} \mathrm{C}$ for $1007 \mathrm{FS}$. All samples were amplified in duplicate and each run included DNA from sequenceverified wild-type, heterozygous and homozygous genotypes for each of the variants. High-resolution melt curve data were analysed using the manufacturer's software. All raw data were normalised by selecting linear regions before and after the melting transition, and the resulting melt curve profiles were compared to melting profiles from known positive control samples included in the same run.

\section{RNA purification and cDNA preparation}

A total of 11 samples from 11 non-disease controls and 95 samples from 65 Crohn's disease patients were subjected to RNA and DNA isolation. Total RNA was isolated from two to three mucosal biopsy specimens (per sample) using AllPrep DNA/RNA Mini kit (OIAGEN, Hilden, Germany) according to the manufacturer's instructions. All extractions included an on-column RNase-free DNase I digestion (OIAGEN) step. As poor RNA quality can affect real-time polymerase chain reaction (RT-PCR) measurements, RNA integrity was assessed by Bioanalyzer (Agilent Technologies, California, USA) and only samples with an RNA integrity number (RIN) $\geqslant 5$ were used for quantitative RT-PCR. ${ }^{26}$ First-strand cDNA synthesis was performed using SuperScript III First-strand cDNA Synthesis SuperMix for qRT-PCR (Invitrogen). Each reaction contained 500 ng total RNA as determined by the Bioanalyzer. The cDNA samples were then diluted 1:20 for use in quantitative RT-PCR.

\section{Real-time quantitative polymerase chain reaction analysis}

Primers were designed using PrimerQuest (Integrated DNA Technologies, Iowa, USA; http://www.idtdna.com/Scitools/ Applications/Primerquest) (table 2). The primer concentrations 
Table 2 Primer sequences and product sizes for real-time polymerase chain reaction

\begin{tabular}{llll}
\hline Target & Forward primer & Reverse primer & Product size (bp) \\
\hline B2M & AGGCTATCCAGCGTACTCCAAAGA & CGGATGGATGAAACCCAGACACAT & 105 \\
P0LR2A & GTGTTCCTTTGAGGAACGGTGGA & TCTGCATCAAGCAGGAGGTCAAAG & 147 \\
RPLPO & GGGCACCATTGAAATCCTGAGTGA & CATTGTCGAACACCTGCTGGATGA & 140 \\
TBP & CCCGAAACGCCGAATATAATCCCA & TGTTCTTCACTCTTGGCTCCTGTG & 127 \\
UBC & GCAGTTCTTGTTGTGGATCGCTG & TGACATTCTCGATGGTGTCACTGG & 119 \\
UBE2D2 & TGGCTCTGAAGAGAATCCACAAGG & TCCACCCTGATAGGGACTGTCATT & 143 \\
DEFA5 & TCCAGGAAAGAGCTGATGAGGCTA & ACCTGAGGTTCTAAGAGCAGAGAGTC & 110 \\
DEFA6 & AAAGCTTATGAGGCTGATGCCCAG & CTTCTGCAATGGCAAGTGAAAGCC & 134 \\
PLA2G2A & GGAAAGGAAGCCGCACTCAGTTAT & CACATCCACGTTTCTCCAGACGTT & 139 \\
LYZ & CCGCTACTGGTGTAATGATGGCAA & TGCTCTAATGCCTTGTGGATCACG & 142 \\
VILI & CATGCGCTGAACTTCATCAAAGCC & CCACTTCTGGAAGAGCTGCTGAAA & 108 \\
IL6 & CATGTGTGAAAGCAGCAAAGAGGC & CACCAGGCAAGTCTCCTCATTGAA & 112 \\
IL8 & CTCTTGGCAGCCTTCCTGATTTCT & GGGTGGAAAGGTTTGAGTATGTCT & 113 \\
\hline
\end{tabular}

B2M, $\beta_{2}$-microglobulin; POLR2A, polymerase (RNA) II (DNA directed) polypeptide A, $220 \mathrm{kDa}$; RPLPO, ribosomal protein, large, PO; TBP, TATA box binding protein; UBC, ubiquitin C; UBE2D2, ubiquitin-conjugating enzyme E2D 2; DEFA5, $\alpha$-defensin 5, Paneth cellspecific; DEFA6, $\alpha$-defensin 6, Paneth cell-specific; PLA2G2A, phospholipase $A_{2}$, group IIA (platelets, synovial fluid); LYZ, lysozyme (renal amyloidosis); VIL1, villin 1; IL6, interleukin 6 (interferon- $\beta 2$ ); IL8, interleukin 8.

were optimised so that the minimum concentrations for each primer pair gave the lowest threshold cycle $\left(\mathrm{C}_{\mathrm{T}}\right)$ and greatest signal magnitude (delta $\mathrm{Rn}$ ) with minimal non-specific amplification. RT-PCR amplification was performed using platinum SYBR Green qPCR SuperMix UDG (Invitrogen) in a total volume of $15 \mu \mathrm{l}$ containing $4 \mu \mathrm{l}$ cDNA (10 ng), $2 \times$ qPCR SuperMix, $300 \mathrm{nmol} / \mathrm{l}$ each primer and $\mathrm{H}_{2} \mathrm{O}$. Reactions were amplified using Rotor-Gene 6000 (Corbett Life Science) and quantified using the manufacturer's software. The thermocycling conditions comprised $3 \mathrm{~min}$ at $95^{\circ} \mathrm{C}$ and then 40 cycles at $95^{\circ} \mathrm{C}$ for $15 \mathrm{~s}, 59^{\circ} \mathrm{C}$ for $15 \mathrm{~s}$, and $72^{\circ} \mathrm{C}$ for $20 \mathrm{~s}$, followed by a standard melt curve analysis to validate the specificity of the PCR products. All samples were amplified in triplicate from the same RNA preparation. A calibrator sample [BD qPCR Human Reference Total RNA (BD Biosciences, Clontech, Palo Alto, CA, USA) for LYZ, PLA2G2A, VIL1, IL6, IL8 or normal ileum RNA for DEFA5 and DEFA6] was included in each run. RT-PCR efficiencies were determined for each gene and relative quantification of target mRNA was carried out using the Pfaffl method. ${ }^{27}$ To further validate our quantitative RT-PCR data, each target gene was normalised separately to two different normalisation genes.

\section{Normalisation gene selection}

We evaluated six housekeeping genes for suitability for normalising quantitative RT-PCR data from ileal Crohn's disease tissue using the BestKeeper software tool. ${ }^{28}$ Raw input data (average replicate $\mathrm{C}_{\mathrm{T}}$ and RT-PCR efficiency values) for each gene were obtained. Normalisation gene candidates were assessed for their expression stability in tissue from the ileum and right colon, and at various stages of disease and inflammation as assessed by a pathologist. The biopsy tissue panel included two non-disease control samples, five non-inflamed Crohn's disease ileum, three mildly inflamed Crohn's disease ileum, three extensively inflamed ileum and two samples from non-inflamed Crohn's disease right colon. The normalisation gene candidates were chosen based on $\mathrm{C}_{\mathrm{T}}$ value and RNA transcription profile stability results from previously published studies. ${ }^{29-31}$ The genes were $\beta_{2}$-microglobulin (B2M; Accession No. NM_004048), RNA polymerase II largest subunit (POLR2A; Accession No. NM_000937), Ribosomal Protein Large P0 (RPLP0; Accession No. NM_001002), Tata-Box binding protein (TBP; Accession No. NM_003194), Ubiquitin C (UBC;
Accession No. NM_021009), and Ubiquitin-conjugating enzyme E2D2 (UBE2D2; Accession No. NM_181838).

\section{Immunohistochemistry}

HD-5 (DEFA5) immunohistochemistry and haematoxylin and eosin (H\&E) histological staining was performed on parallel sections of ileal Crohn's disease resection tissue. For HD-5 immunohistochemistry, paraffin sections from non-inflamed and inflamed ileal tissue $(4 \mu \mathrm{m})$ were affixed to Superfrost Plus adhesive slides (Menzel-Gläser; Braunschweig, Germany) and air-dried overnight at $45^{\circ} \mathrm{C}$. After dewaxing in xylol and rehydration through descending graded alcohols to distilled $\mathrm{H}_{2} \mathrm{O}$, sections were transferred to Target Retrieval Solution pH 9.0 (Dako, Carpinteria, CA, USA), and antigen retrieval was performed for $7 \mathrm{~min}$ at $121^{\circ} \mathrm{C}$ using a commercial pressure cooker (Decloaker; Biocare Medical, Walnut Creek, CA, USA). The sections were cooled and transferred to Tris-buffered saline with Tween-20 (0.05 mol/1 Tris, $0.15 \mathrm{~mol} / 1 \mathrm{NaCl}, 0.1 \%$ Tween20) (TBS-T), pH 7.2-7.4. The subsequent immunohistochemical protocol was performed on an automated immunostainer (AutoStainer; Dako). Endogenous peroxidase activity was quenched by incubating the sections with $1.0 \% \mathrm{H}_{2} \mathrm{O}_{2}$ in TBS for $10 \mathrm{~min}$. Following washing in two changes of TBS-T, nonspecific antibody binding was inhibited by incubating the sections with Eraser protein block (BioCare Medical) for $10 \mathrm{~min}$. Excess blocking reagent was blown from the sections and the primary antibody (rabbit anti-human HD-5 serum, kindly provided by E.M. Porter, Department of Biological Sciences California State University Los Angeles, CA) applied at 1/12 000 dilution in commercial antibody diluent (Dako) for $90 \mathrm{~min}$. Following this and subsequent incubations the sections were washed in two changes of TBS-T. Sections were then incubated with MACH3 Rabbit Probe ${ }^{\mathrm{TM}^{\mathrm{TM}}}$ (Biocare Medical) for $20 \mathrm{~min}$, then MACH3 R-Polymer-HRP ${ }^{+\mathrm{TM}}$ (BioCare Medical) for $10 \mathrm{~min}$. Colour was developed using Liquid DAB+ substrate chromogen system $^{\mathrm{TM}}$ (Dako) for 6-8 min. Then sections were washed in running tap water, lightly counterstained in Mayer's haematoxylin, dehydrated through ascending graded alcohols, cleared in xylene, and mounted using DePeX (BDH Gurr, Poole, UK).

\section{Statistical analysis}

The experimental unit tested was gene expression as quantified via RT-PCR and normalised by the Pfaffl method. ${ }^{23}$ Expression 
Figure 1 lleal mRNA expression of Paneth cell antimicrobial molecules $(A) \alpha$ defensin 5 (DEFA5), (B) lysozyme (LYZ), (C) $\alpha$-defensin 6 (DEFA6), (D) phospholipase- $A_{2}$ (PLA2G2A), (E) the epithelial cell marker, villin; and the proinflammatory cytokines (F) interleukin 6 (IL6) and (G) interluekin 8 (IL8) in healthy non-disease controls (Con), and Crohn's disease patients with non-inflamed (NI) and inflamed (I) ileum with respect to NOD2 mutation status. Data normalised to B2M. Wt, NOD2 wild-type; Mut, NOD2 mutation. ${ }^{* *} p<0.001,{ }^{*} p<0.05$, t $p>0.1$.
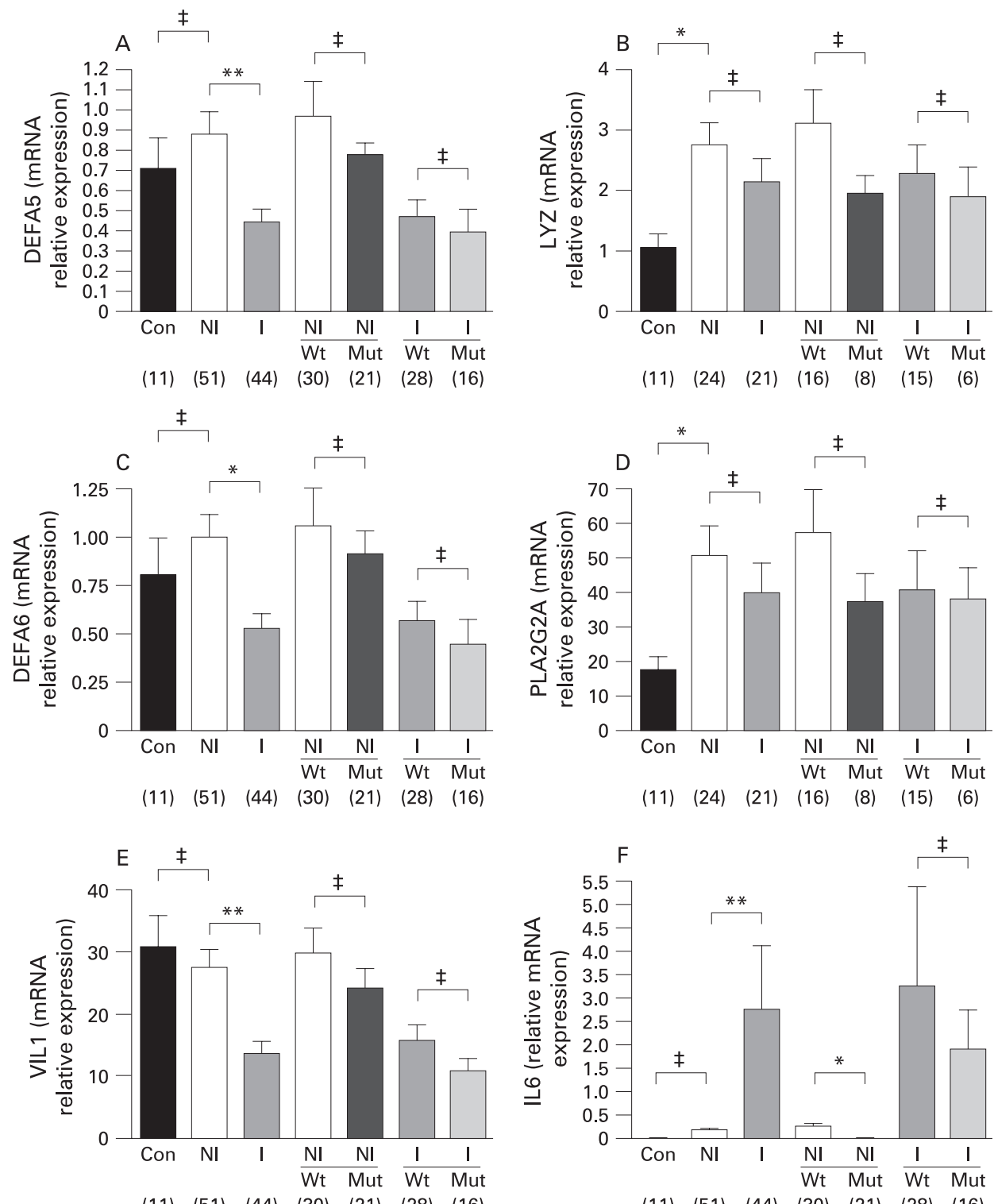

$\begin{array}{llllll}\text { (11) } & \text { (51) (44) (30) (21) (28) (16) }\end{array}$

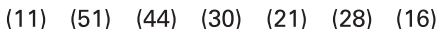

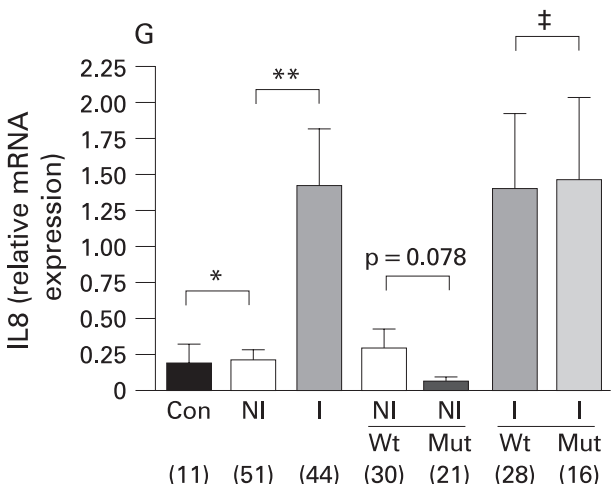

data were analysed using the parametric independent samples ttest and analysis of covariance (ANCOVA) after log transformation. Expression data from IL6 and IL8 did not fit the parametric assumptions; hence the non-parametric MannWhitney U test was performed here. Pearson's correlation analysis was utilised to quantify the expression associations between the genes of interest. $p$-Values $<0.05$ were considered statistically significant. All statistical analysis was performed using SPSS for Windows (version 15.0).

\section{RESULTS}

\section{NOD2 genotyping}

NOD2 genotyping was performed via high-resolution melt curve analysis. Of the 11 non-disease controls in our study, three carried a NOD2 variant (one heterozygote for R702W, one heterozygote for G908R, and one compound heterozygote for R702W and G908R). Of the 65 Crohn's disease patients, 27 (42\%) possessed a NOD2 variant (nine were heterozygous for R702W, four were heterozygous for G908R, one was heterozygous for 1007fs, seven 


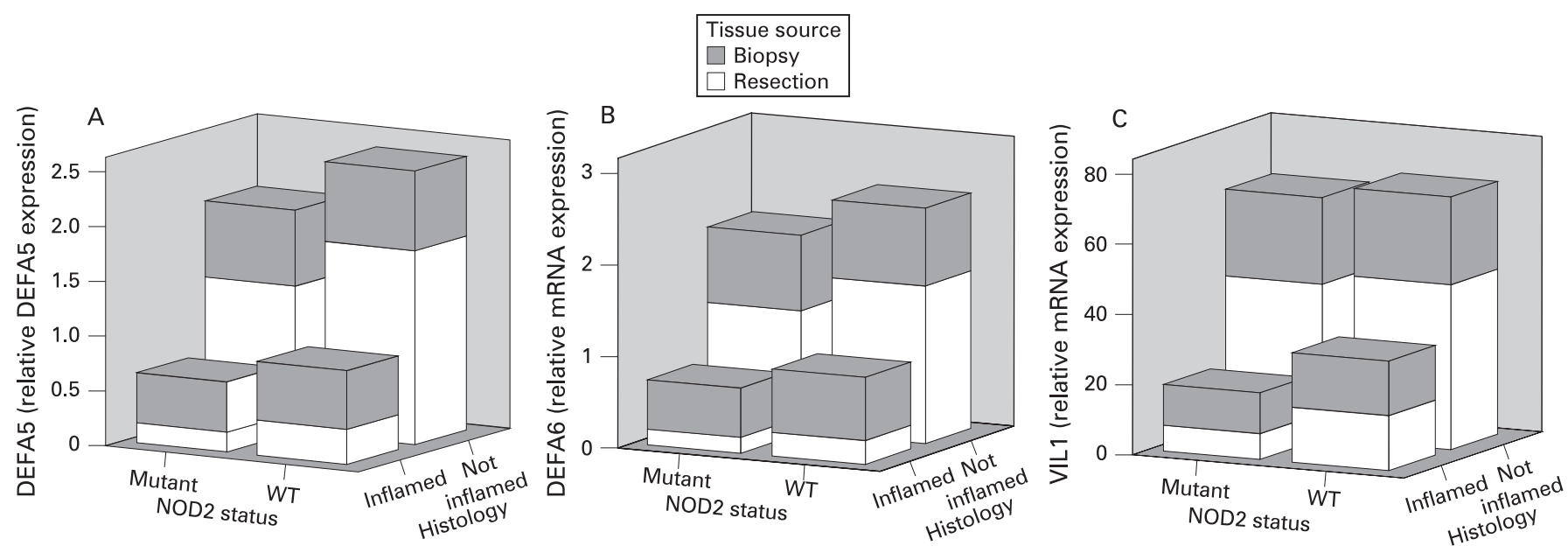

Figure 2 Three-dimensional plots showing the effects of inflammation (defined according to histology) and NOD2 mutation status on mRNA expressions for the genes (A) $\alpha$-defensin 5 (DEFA5), (B) $\alpha$-defensin 6 (DEFA6), and (C) vilin (VIL1).

were compound heterozygotes (five for R702W/1007fs and two for G908R/1007fs) and six were homozygous mutants (three for R702W and three for 1007fs). We also investigated the frequency of the P268S variant in our control and case populations. The allele frequencies of this variant were as follows: controls - wildtype, two; heterozygous, eight; homozygous, one; and, Crohn's disease - wild-type, 25 (39\%); heterozygous, 23 (35\%), homozygous, $17(26 \%)$. In all cases, patients who were wild-type for the
P268S variant were wild-type for R702W, G908R and 1007fs. Furthermore, all six patients homozygous for either R702W or $1007 \mathrm{fs}$ also possessed a homozygous P268S variant. These data indicate that the P268S variant can be a useful predictor for the common NOD2 genotype; wild-type P268S genotype indicates a wild-type genotype result for R702W, G908R and 1007fs; and a homozygous P268S variant flags individuals homozygous for R702W, G908R or 1007fs. These findings in the ileal tissue patient
Figure 3 Representative haematoxylin and eosin (H\&E)-stained and anti-HD- $5(\alpha-$ defensin 5, DEFA5)-stained non-inflamed $(\mathrm{A}-\mathrm{H})$ and inflamed (I-P) ileal tissue sections from a NOD2 wild-type and a NOD2-mutant Crohn's disease (CD) patient. Images were scanned at $\times 40$ magnification using an automated imaging analysis system (ScanScope T2 System, Aperio Technologies, Vista, California, USA). $\times 1$ and $\times 8$ digital zooms were applied for the images presented ( $\times 1$ zoom: $A, C, E, G, I, K, M$, 0 ; and $\times 8$ zoom: $B, D, F, H, J, L, N, P)$. The solid arrows point to Paneth cells present in the base of the epithelial crypt cells, visualised by H\&E staining (red) and anti-HD-5 staining (dark brown). Note the low density of Paneth cells in inflamed mucosa in both NOD2-wild-type and NOD2-mutant Crohn's disease ileum ( $M$ and $0, \times 1$ digital zoom). In some segments, there is a complete absence of both epithelial cells and Paneth cells (white arrow heads).

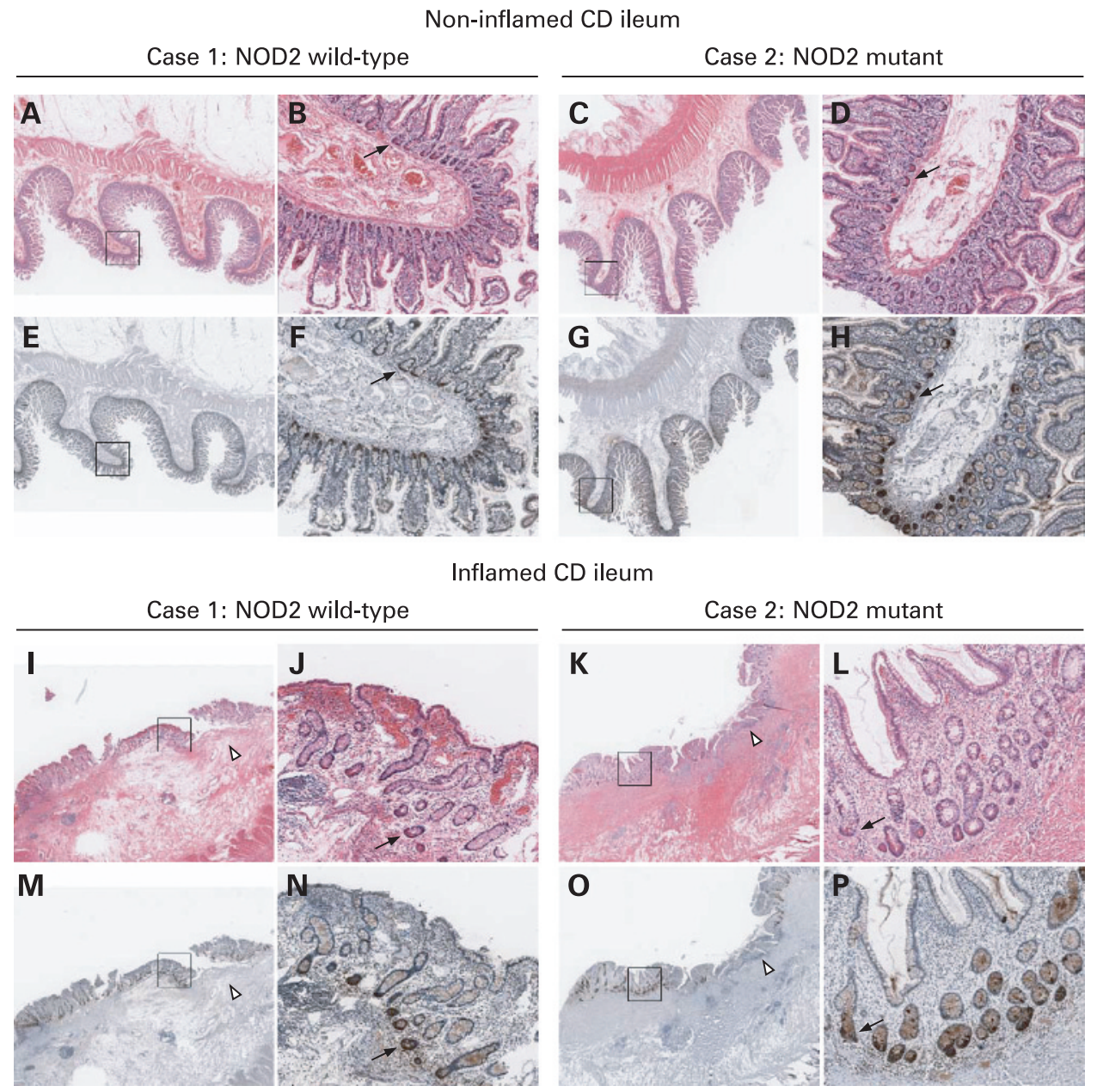


group were confirmed in our large Crohn's disease case cohort (data not shown), and also agrees with the P268S variant distribution previously described. ${ }^{32}$

\section{Selection of normalisation gene candidates}

Prior to performing mRNA expression analysis of our target genes in ileal control and Crohn's disease biopsy tissue, we evaluated the stability of expression of six housekeeping genes across a range of conditions and disease states (location, ileum/ right colon; inflammation, no inflammation/mild inflammation/active or extensive inflammation; and disease states, nondisease control/ileal Crohn's disease) using BestKeeper. Ranked from the most stable to the least stable were B2M, RPLP0, TBP, UDE2D2, UBC and POLR2A. We selected B2M and RPLP0 as the normalising genes in this study.

\section{DEFA5 and DEFA6 expression correlates with inflammatory state and not NOD2 mutation status}

In Crohn's disease patients, we detected a significant reduction in DEFA5 and DEFA6 mRNA expression in histologically confirmed inflamed mucosa $(n=44)$ compared to non-inflamed ileal mucosa $(n=51) \quad(p<0.001$ and $p<0.05$, respectively) (fig 1A,C). The associations remained significant after adjustment for confounders including drug treatment (treatment vs no treatment), sample type (resection vs biopsy), and NOD2 status (wild-type vs mutant) (DEFA5, $p<0.001$; DEFA6, $\mathrm{p}<0.05)$. No association was found between NOD2 status (R702W, G908R and 1007fs), and the mRNA expression levels of DEFA5 and DEFA6, nor could we find a significant association between the variant P268S, a marker of NOD2 haplotype, and $\alpha$-defensin expression (data not shown).

In our study, mRNA expression of VIL1, a marker of epithelial cell content, was significantly decreased in inflamed ileal mucosa $(p<0.001$, after adjustment for confounders). We noticed a marked similarity between the results shown in fig 1A,C and those in fig 1E, representing mRNA expressions for DEFA5, DEFA6 and VIL1, respectively, across all sample groups (controls and Crohn's disease patients with and without NOD2 stratification). To further explore the relationship between $\alpha$ defensin expression and VIL1 mRNA expression in Crohn's disease ileum, we generated three-dimensional plots comparing DEFA5, DEFA6 and VIL1 mRNA expression levels (grouped by sample type; biopsy, resection) with NOD2 status and inflammation (as defined histologically) (fig 2). Together, fig 1A,C,E and fig 2 demonstrate that, regardless of sample type, inflammation is the primary factor influencing expression, and not NOD2 mutation status.

In support of this observed association between inflammation and $\alpha$-defensin expression, we identified a positive correlation between the mRNA levels of DEFA5 and VIL1, in that as VIL1 mRNA expression levels increased, DEFA5 mRNA expression levels also increased $(p<0.0001)$.

Anti-HD-5 and H\&E immunohistochemical staining was performed on parallel sections from non-inflamed and inflamed NOD2 wild-type and NOD2 mutant ileal Crohn's disease tissue. Representative anti-HD-5 immunostaining is presented in fig 3. Regardless of NOD2 genotype, HD-5 protein expression was reduced in inflamed ileal tissue. This specific reduction of $\mathrm{HD}-5$ protein expression in inflamed ileum appears due to the loss of surface epithelial cells and reduced Paneth cell numbers (visible by $\mathrm{H} \& \mathrm{E}$ staining (red), I-L; and anti-HD-5 staining (dark brown), M-P), as a result of inflammatory changes. Of note, HD-5 staining intensity is similar between NOD2 wild-type and NOD2 mutant cases in both non-inflamed and inflamed tissue sections.

In the small intestine, PLA2G2A is specifically expressed by Paneth cells and hence PLA2G2A expression levels reflect Paneth cell numbers. ${ }^{33}$ As further confirmation of the link between Paneth cell density and $\alpha$-defensin expression, we performed a simple correlation analysis of the data (fig 4). For all samples ( $n=106$; controls, non-inflamed and inflamed samples) irrespective of NOD2 status, there was a strong positive relationship between DEFA5 and PLA2G2A mRNA levels $(r=0.77, p<0.001)$ and between DEFA6 and PLA2G2A mRNA levels $(r=0.76$, $p<0.001)$. The strongest correlations were seen in the noninflamed sample group (solid line in fig 4, panels A and B) (DEFA5 and PLA2G2A, $r=0.91$; DEFA6 and PLA2G2A, $r=0.97$ ).

As a small proportion of patients contributed two biopsies for gene expression analysis, we tested the within-individual and between-individual variance to rule out any potential sample bias. We found no difference in gene expression dependent upon whether the samples were from those patients who contributed one biopsy compared to those who contributed more than one.

\section{LYZ and PLA2G2A mRNA expression is increased in Crohn's disease mucosa}

There was no significant difference in $\mathrm{mRNA}$ expression levels for LYZ or PLA2G2A in inflamed $(\mathrm{n}=21)$ and non-inflamed
Figure 4 Fitted line plots comparing (A) $\alpha$-defensin 5 (DEFA5) and phospholipase- $A_{2}$ (PLA2G2A) mRNA levels and (B) $\alpha$-defensin 6 (DEFA6) and PLA2G2A mRNA levels.
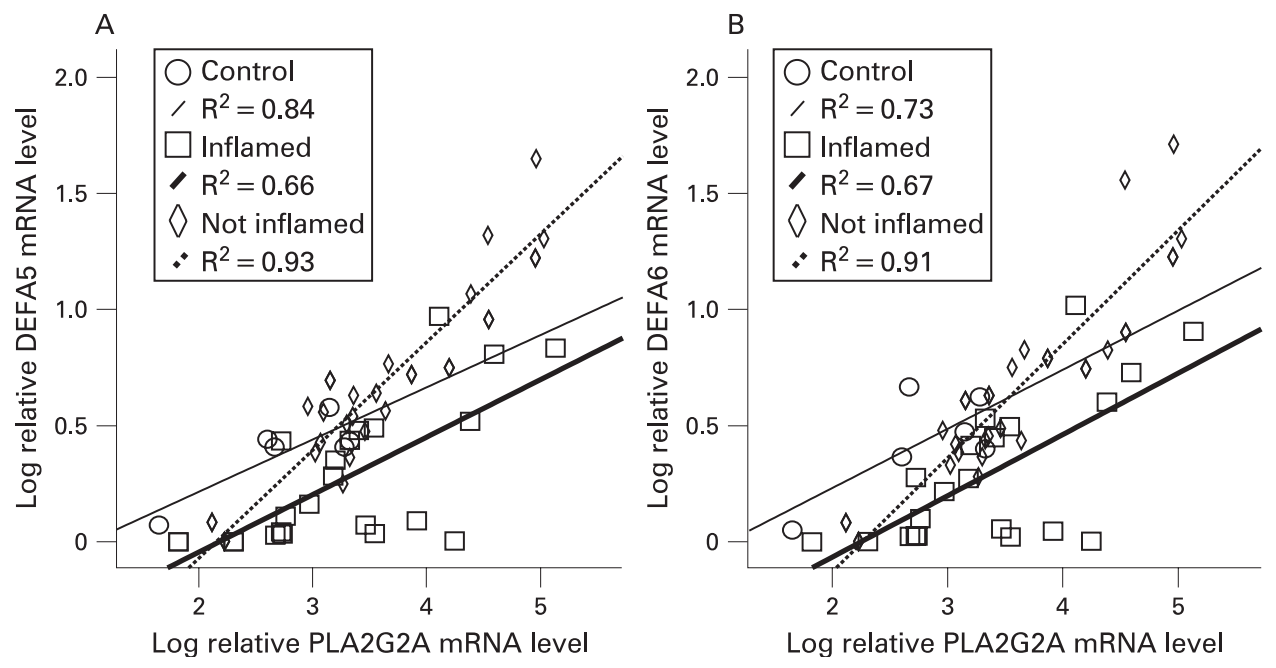
Crohn's disease mucosa $(n=24)$, nor was the expression of these Paneth cell antimicrobials influenced by NOD2 genotype. We did, however, detect a significant increase in LYZ and PLA2G2A mRNA expression levels in non-inflamed Crohn's disease mucosa compared to controls $(p=0.024$ and $p=0.022$, respectively) (fig $1 \mathrm{~B}, \mathrm{D})$. As no significant association was detected between LYZ and PLA2G2A expression, inflammation and NOD2 status in a subgroup of ileal Crohn's disease tissue samples (non-inflamed $n=24$; inflamed $n=21$ ), the expressions of these genes were not evaluated further in a second set of ileal Crohn's disease samples (non-inflamed, $\mathrm{n}=27$; inflamed, $\mathrm{n}=23)$.

\section{IL6 and IL8 mRNA expression is associated with inflammatory state and is influenced by NOD2 mutation status (R702W, G908R, 1007fs)}

Expression levels of the inflammatory cytokines IL6 and IL8 were significantly increased in inflamed ileal mucosa $(p<0.001$ for both) (fig 1F,G). Consistent with these data, we detected negative correlations between the expression levels of VIL1, a marker of epithelial cell content, and both inflammatory cytokines $(p<0.01$, data not shown). The expression of both cytokines in non-inflamed ileum, but not inflamed ileum, was decreased in a NOD2 variant background $(p=0.033$ and $p=0.078$, respectively). There was a small but significant increase in IL8 expression levels in non-inflamed ileal mucosa from Crohn's disease patients versus controls $(p=0.017)$.

\section{DISCUSSION}

$\alpha$-Defensins are potent antimicrobial peptides produced by Paneth cells and have a major role in the innate immunity of the small intestine. Two studies by Wehkamp et al ${ }^{18}{ }^{19}$ report a decrease in $\boldsymbol{\alpha}$-defensin expression in inflamed ileal mucosa from Crohn's disease patients, with a more pronounced decrease in Crohn's disease patients carrying NOD2 mutations. In agreement with Wehkamp et al, ${ }^{18}$ we find DEFA5 and DEFA6 expression is reduced in inflamed ileal mucosa compared to noninflamed ileal mucosa whereas the expressions of antimicrobials, LYZ and PLA2G2A, does not differ significantly between Crohn's disease sample groups. These associations did not change after adjustment for confounders such as medical treatment, type of sample and NOD2 status. However, the association between reduced $\alpha$-defensin expression and NOD2 mutation status could not be reproduced in our study. Instead, we find that $\alpha$-defensin expression is dependent on the inflammatory state of the sample (defined according to VIL1 mRNA expression level and histological examination) and not NOD2 genotype (R702W, G908R, 1007fs). Our HD-5 immunohistochemical studies of ileal Crohn's disease tissue complements results obtained from these RNA data. We could also find no association between $\alpha$-defensin expression and P268S variant status. It is worth noting, however, that neither of the earlier studies $^{18}{ }^{19}$ examined $\boldsymbol{\alpha}$-defensin levels in samples from unaffected (non-inflamed) ileal mucosa from Crohn's disease patients carrying a NOD2 mutation. Hence, the association between defensin expression and NOD2 status in both these studies can only be said to occur in affected (inflamed or Crohn's disease ileitis) tissue. The discrepancy between our study and the studies by Wehkamp et al ${ }^{18} 19$ may be due to differences in study design. For example, our data are based on a larger sample size, our tissue cohort included non-inflamed samples from Crohn's disease patients carrying a NOD2 mutation, and all ileal Crohn's disease tissue samples were classified as either non-inflamed or inflamed according to histological findings and not macroscopic classification. The key observations in our study come from the non-inflamed samples, where there is no significant tissue damage and associated secondary effects due to the inflammation itself. We postulate that if a defect in $\alpha$-defensin production is the underlying/primary cause of ileal Crohn's disease, abnormal $\alpha$ defensin levels would be evident in non-inflamed ileal mucosa prior to the development of inflammatory lesions. Our results suggest that low $\alpha$-defensin mRNA levels in ileal tissue from Crohn's disease patients, irrespective of NOD2 genotype, is most likely due to the loss of epithelium as a consequence of inflammatory changes. Underpinning this hypothesis is our demonstration of a strong positive relationship between mRNA levels of PLA2G2A, a marker of Paneth cell mass, and the $\alpha-$ defensins. The present study data lend support to the importance of mucosal healing in Crohn's disease patients. Mucosal healing in Crohn's disease is associated with a reduction in the inflammation, ${ }^{34}$ and we speculate a corresponding increase or restoration of $\alpha$-defensin production due to regeneration of epithelial cells in the intestinal mucosa. In support of this hypothesis, we find a positive correlation between VIL1 and DEFA5 mRNA expression levels, and similar levels of $\alpha$-defensins in non-inflamed ileal samples from Crohn's disease patients (NOD2 wild-type and NOD2 mutant) and in non-disease controls. Wehkamp et al ${ }^{18}$ also detected comparable $\alpha$-defensin levels in non-inflamed ileal samples from healthy controls and NOD2 wild-type Crohn's disease patients.

In the present study, we detected significant increases in the mRNA expression levels of the Paneth cell antimicrobials LYZ and PLA2G2A in non-inflamed Crohn's disease mucosa compared to non-inflamed control mucosa. We recognise, however, that these data may be subject to error due to the relatively small sample size of the control group $(n=11)$ and/or by the contribution made by other cell types of the small intestine to LYZ and PLA2G2A expression. ${ }^{6}{ }^{35}$

Consistent with previous observations in peripheral blood mononuclear cells, ${ }^{20} 216$ we detected reduced IL8 mRNA expression in those patients carrying variant NOD2, supporting a defect in innate immune responses mediated via pattern recognition receptors in Crohn's disease. Additionally, we report for the first time, reduced IL6 expression in ileal mucosa in Crohn's disease patients carrying a NOD2 mutation, suggesting that this primary genetic abnormality may be associated with a more generalised defect in the mucosal immune response to the local microbial flora.

\section{CONCLUSION}

The view that the primary factor in ileal Crohn's disease pathogenesis is due to low $\alpha$-defensin production $^{18} 19$ is not supported by our study findings. Rather, we find that the reduction in $\alpha$-defensin expression is dependent on the inflammatory state of the tissue and is therefore likely to be due to loss of epithelium as a consequence of secondary changes rather than being the inciting event prior to inflammation. Once the disease is manifested, the small intestinal crypts would presumably be susceptible to bacterial invasion due to low levels of antimicrobial peptides, including the $\alpha$-defensins, as a result of epithelial cell loss. The invading bacteria would then be set to provoke further inflammatory responses in the small intestinal mucosa.

Acknowledgements: The authors would like to thank Assistant Professor Edith Porter (Department of Biological Sciences, California State University, USA) for the kind gift of anti-HD-5 serum, Professors Michael McGuckin (Mater Medical Research 
Institute, Brisbane, Australia) and Peter O'Rourke (Queensland Institute of Medical Research, Brisbane, Australia) for providing critical reviews of the manuscript; the Royal Brisbane and Women's Hospital Research Foundation and the Australian Crohn's and Colitis Association for funding the purchase of the real-time PCR machine; all the participants taking part in this research; and Mrs Susan Mason, IBD nurse at the Royal Brisbane and Women's Hospital, for help with sample collection.

Funding: EVF was partly funded by a Reginald Ferguson Research Fellowship from The University of Queensland; GLR-S is supported by a Queensland Government SmartState Clinical Research Fellowship, and by a Practitioner Fellowship from the Royal Brisbane and Women's Hospital Research Foundation.

Competing interests: None.

Ethics approval: This study was approved by the Human Research Ethics Committees of the Royal Brisbane and Women's Hospital and the Queensland Institute of Medical Research, 25 August 2004.

\section{REFERENCES}

1. Bonen DK, Cho JH. The genetics of inflammatory bowel disease. Gastroenterology 2003; 124:521-36.

2. Sawczenko A, Sandhu BK, Logan RF, et al. Prospective survey of childhood inflammatory bowel disease in the British Isles. Lancet 2001;357:1093-4.

3. Yang SK, Loftus EV Jr, Sandborn WJ. Epidemiology of inflammatory bowel disease in Asia. Inflamm Bowel Dis 2001;7:260-70.

4. Radford-Smith G, Pandeya N. Associations between NOD2/CARD15 genotype and phenotype in Crohn's disease - Are we there yet? World J Gastroenterol 2006;12:7097-103

5. Simon GL, Gorbach SL. Normal alimentary tract flora. New York: Raven Press, 1995.

6. Elphick DA, Mahida YR. Paneth cells: their role in innate immunity and inflammatory disease. Gut 2005:54:1802-9.

7. Wehkamp J, Chu H, Shen B, et al. Paneth cell antimicrobial peptides: topographical distribution and quantification in human gastrointestinal tissues. FEBS Letters 2006:580:5344-50

8. Ayabe T, Satchell DP, Wilson CL, et al. Secretion of microbial $\alpha$-defensins by intestinal Paneth cells in response to bacteria. Nature Immunology 2000:1:113-8.

9. Porter EM, van Dam E, Valore EV, et al. Broad-spectrum antimicrobial activity of human intestinal defensin 5. Infect Immun 1997;65:2396-401.

10. Ghosh D, Porter E, Shen B, et al. Paneth cell trypsin is the processing enzyme for human defensin-5. Nature Immunology 2002;3:583-90.

11. Ayabe T, Wulff H, Darmoul D, et al. Modulation of mouse Paneth cell alpha-defensin secretion by mlKCa1, a $\mathrm{Ca}^{2+}$-activated, intermediate conductance potassium channel. J Biol Chem 2002;277:3793-800.

12. Salzman NH, Ghosh D, Huttner KM, et al. Protection against enteric salmonellosis in transgenic mice expressing a human intestinal defensin. Nature 2003;422:522-6.

13. Wilson CL, Ouellette AJ, Satchell DP, et al. Regulation of intestinal alpha-defensin activation by the metalloproteinase matrilysin in innate host defense. Science 1999;286:113-7.

14. Inohara N, Ogura $Y$, Fontalba A, et al. Host recognition of bacterial muramyl dipeptide mediated through NOD2. J Biol Chem 2003:278:5509-12.

15. Eckmann L, Karin M. NOD2 and Crohn's disease: loss or gain of function? Immunity 2005;22:661-7.
16. Lala S, Ogura Y, Osborne $C$, et al. Crohn's disease and the NOD2 gene: a role for paneth cells. Gastroenterology 2003;125:47-57.

17. Ahmad T, Armuzzi A, Bunce M, et al. The molecular classification of the clinical manifestations of Crohn's disease. Gastroenterology 2002;122:854-66.

18. Wehkamp J, Harder J, Weichenthal M, et al. NOD2 (CARD15) mutations in Crohn's disease are associated with diminished mucosal alpha-defensin expression. Gut 2004;53:1658-64.

19. Wehkamp J, Salzman NH, Porter E, et al. Reduced Paneth cell alpha-defensins in ileal Crohn's disease. Proc Natl Acad Sci USA 2005:102:18129-34.

20. Li J, Moran T, Swanson E, et al. Regulation of IL8 and IL1 $\beta$ expression in Crohn's disease associated NOD2/CARD15 mutations. Hum Mol Genet 2004;13:1715-25.

21. van Heel DA, Ghosh S, Butler M, et al. Muramyl dipeptide and toll-like receptor sensitivity in NOD2-associated Crohn's disease. Lancet 2005;365:1794-6.

22. Lennard-Jones JE. Classification of inflammatory bowel disease. Scand J Gastroenterol 1989;170:2-6.

23. Hugot JP, Chamaillard M, Zouali $\mathrm{H}$, et al. Association of NOD2 leucine-rich repeat variants with susceptibility to Crohn's disease. Nature 2001;411:599-603.

24. Ogura Y, Bonen DK, Inohara N, et al. A frameshift mutation in NOD2 associated with susceptibility to Crohn's disease. Nature 2001:411:603-6.

25. Bonen DK, Ogura Y, Nicolae DL, et al. Crohn's disease-associated NOD2 variants share a signaling defect in response to lipopolysaccharide and peptidogylcan. Gastroenterology 2003;124:140-6.

26. Fleige $\mathbf{S}$, Pfaffl MW. RNA integrity and the effect on the real-time qRT-PCR performance. Mol Aspects Med 2006;27:126-39.

27. Pfaffl MW. A new mathematical model for relative quantification in real-time RTPCR. Nucl Acids Res 2001;29:e45.

28. Pfaffl MW, Tichopad A, Prgomet C, et al. Determination of stable housekeeping genes, differentially regulated target genes and sample integrity: BestKeeper - Excelbased tool using pair-wise correlations. Biotechnol Lett 2004;26:509-15.

29. Andersen CL, Jensen JL, Ørntoft TF. Normalization of real-time quantitative reverse transcription-PCR data: a model-based variance estimation approach to identify genes suited for normalization, applied to bladder and colon cancer data sets. Cancer Res 2004;64:5245-50.

30. Dheda K, Huggett JF, Bustin SA, et al. Validation of housekeeping genes for normalizing RNA expression in real-time PCR. Biotechniques 2004;37:112-4.

31. Dydensborg AB, Herring E, Auclair J, et al. Normalizing genes for quantitative RTPCR in differentiating human intestinal epithelial cells and adenocarcinomas of the colon. Am J Physiol, Gastrointest Liver Physiol 2006;290:G1067-74.

32. Roberts RL, Gearry RB, Barclay ML, et al. Rapid detection of common CARD15 variants in patients with inflammatory bowel disease. Mol Diagn 2004;8:101-5.

33. Garabedian EM, Roberts LJJ, McNevin MS, et al. Examining the role of Paneth cells in the small intestine by lineage ablation in transgenic mice. J Biol Chem 1997;272:23729-40.

34. D'Haens G, van Deventer S, van Hogezand R, et al. Endoscopic and histological healing with infliximab anti-tumor necrosis factor antibodies in Crohn's disease: a European multicenter trial. Gastroenterology 1999;116:1029-34.

35. Lilja I, Gustafson-Svard C, Franzen L, et al. Presence of group lla secretory phospholipase $A_{2}$ in mast cells and macrophages in normal ileal submucosa and in Crohn's disease. Clin Chem Lab Med 2000;38:1231-6.

36. Marks DJB, Harbord MWN, MacAllister R, et al. Defective acute inflammation in Crohn's disease: a clinical investigation. Lancet 2006;367:668-78. 